\title{
A Personalidade na Tomada de Decisão com o Iowa Gambling Task: Uma Revisão Integrativa
}

\author{
Rui Paixão ${ }^{1}$ \\ Universidade de Coimbra \\ Graça Areias \\ Unidade de Desabituação de Coimbra
}

\begin{abstract}
RESUMO - O Iowa GamblingTask (IGT), apesar de amplamente utilizado na avaliação da tomada de decisão, apresenta uma grande variabilidade nos resultados. Pretende-se com este trabalho proceder a uma revisão integrativa da literatura que relacione a personalidade com o desempenho no IGT, de modo a identificar o papel dessa variável nesse desempenho. Para o efeito, foram selecionados e analisados 74 estudos referenciados na Web of Science e na b-on. Os resultados evidenciam alguma inconsistência e algumas relações não lineares entre variáveis, embora o mau desempenho no IGT surja associado mais frequentemente à dimensão neuroticismo, ansiedade, impulsividade, variáveis socioeconômicas e ao fator antissociabilidade na psicopatia e dependência de substâncias. À luz desses resultados, sugerem-se algumas orientações para a pesquisa na área.
\end{abstract}

Palavras-chave: tomada de decisão, comportamento de risco, personalidade, impulsividade, personalidade antissocial

\section{Personality in Decision Making with the Iowa Gambling Task: An Integrative Review}

\begin{abstract}
Although the Iowa Gambling Task (IGT) has been widely used in evaluation of decision making, there is a large variability in the results on the task. The aim of this study is to perform an integrative review relating personality with IGT performance, in order to clarify the role of personality in this variability. For this purpose 74 studies referenced in Web of Science and the b-on in June 2016 were analyzed.The results show some inconsistency anda nonlinear association with some variables, although poor performance on IGT is more often associated with neuroticism, anxiety, impulsivity, socioeconomic variables and with the anti-sociability factor in psychopathy and substance abuse. In light of these results, orientations for future research are suggested.
\end{abstract}

Keywords: decision making, risk taking, personality, impulsiveness, antisocial personality

O Iowa Gambling Task (IGT; Bechara, Damasio, Damasio, \& Anderson, 1994) é um jogo em que o sujeito tem de escolher cartas de quatro baralhos (A, B, C, D) com o objetivo de ganhar o máximo dinheiro possível ou evitar perder. Sempre que o indivíduo escolhe uma carta, recebe uma recompensa monetária, mas, para algumas cartas, as recompensas são acompanhadas de uma perda variável de dinheiro, estando essas sequências de recompensas e punições pré-definidas. Os baralhos A e B proporcionam as recompensas mais altas, mas também as punições mais elevadas e são "desvantajosos” (Bechara et al., 1994) porque, a longo prazo, as penalizações monetárias ultrapassam as recompensas. Os baralhos $\mathrm{C}$ e $\mathrm{D}$ proporcionam recompensas mais baixas, mas também produzem perdas significativamente mais leves e são "vantajosos" (Bechara et al., 1994) porque, a longo prazo, as recompensas ultrapassam as penalizações.

A tarefa tem sido amplamente utilizada na avaliação da tomada de decisão em condições de incerteza e de risco. Considera-se que a incerteza remete para o desconhecimento das consequências da decisão (nos primeiros ensaios da tarefa), enquanto o risco (nos ensaios finais) se refere a um

1 Endereço para correspondência: Unidade de Desabituação de Coimbra, Administração Regional de Saúde do Centro Pavilhão, n. 12, Hospital Sobral Cid, Conraria, Coimbra, Portugal. CEP: 3.040-714. E-mail: rpaixao@fpce.uc.pt conhecimento probabilístico associado às consequências dessa decisão.

Apesar de amplamente utilizado na avaliação da tomada de decisão, o IGT apresenta desde os estudos iniciais (Bechara \& Damasio, 2002), uma larga variabilidade no desempenho do grupo controle, com $37 \%$ dos sujeitos a exibir desempenho desvantajoso. Esses resultados são suportados por estudos que mostram uma ausência de homogeneidade nos sujeitos da população geral, com elevada percentagem de desempenho pobre e ausência de aprendizagem, assim como uma preferência pelo critério baixa frequência de punição (baralhos B e D) em detrimento das quantias de dinheiro ganho ou perdido (e.g., Franken \& Muris, 2005; Steingroever, Wetzels, Horstmann, Neumann, \& Wagenmakers, 2013).

Considerando a importância que tem sido atribuída aos modelos de personalidade na justificação da variância observada nos resultados com o IGT, este trabalho visa proceder à revisão integrativa dos estudos que averiguaram o papel dessa variável na tomada de decisão com o IGT. Os critérios de exclusão envolveram os estudos de avaliação farmacológica e psicoterapêutica, os estudos com populações clínicas, nomeadamente perturbações do Eixo I da DSM IV TR (American Psychiatric Association, 2000) e diferentes tipos de perturbações neurológicas. No entanto, alguns dos primeiros estudos de pacientes lesionados pré-frontais ventromediais foram referenciados, dada a sua importância 
histórica para a tarefa. Os estudos sobre tomada de decisão realizados com tarefas diferentes (e. g., Columbia Card Task, Wisconsin Card Sort Task; Balloon Analogue Risk Task; Game of Dice Task) foram também excluídos.

Os critérios de inclusão envolveram os estudos com o IGT sobre as diferentes variáveis de personalidade, incluindo perturbações da personalidade antissocial (e psicopatia), perturbações da personalidade borderline, comportamentos aditivos, comportamentos de risco, impulsividade e ansiedade. A inclusão dessas condições teve como fundamento a sua importância e articulação dimensional com as características de personalidade. Finalmente, um último critério privilegiou a atualidade dos estudos.

As pesquisas foram realizadas na Online Knowledge Library (b-on) e na Web of Science (anteriormente designada como Web of Knowledge), considerando os trabalhos publicados até junho de 2016. Essa seleção teve como fundamento a extensão e importância científica dessas bases: $a$ b-on porque garante o acesso às mais importantes publicações periódicas científicas internacionais e a $\mathrm{Web}$ of Science porque é atualmente a primeira plataforma de pesquisa científica. Essa última plataforma agrega as bases de dados também conhecidas como Science Citation Indexes e inclui bases de referência bibliográfica, com indicadores de citação a partir dos quais é calculado, entre outros indicadores, o fator de impacto das publicações periódicas.

As pesquisas realizadas nessas plataformas utilizaram os descritores Iowa Gambling Task AND personality, tendo sido identificados, respectivamente, 385 e 334 resultados. Em uma primeira fase, esses trabalhos foram analisados considerando a informação disponível no abstract, particularmente o objetivo do estudo, o método utilizado e as principais conclusões. Essa análise permitiu uma seleção de 138 trabalhos. A análise da versão integral desses trabalhos permitiu uma segunda seleção, privilegiando a atualidade da publicação e a integração de resultados de pesquisas anteriores. Dessa segunda seleção restaram 74 trabalhos, dos quais três são revisões (incluindo um livro) e 71, estudos experimentais com grupos de controle. Os trabalhos experimentais foram classificados de acordo com as seguintes categorias: teorias e fatores de personalidade, inteligência cognitiva e emocional, idade e desenvolvimento, ansiedade, afetos positivos e negativos, impulsividade, sensibilidade à recompensa e à perda, procura de sensações, adições, psicopatia e comportamentos antissociais, comportamentos borderlines, comportamentos de risco (incluindo jogo patológico) e variáveis socioeconômicas. A maioria dos artigos pontuou simultaneamente em duas ou três dessas categorias.

\section{Resultados e Discussão}

\section{IGT e Personalidade}

No que respeita à personalidade destacam-se os estudos com o modelo big five (Costa \& McCrae, 1992) e com o modelo dos três fatores de Eysenck (Eysenck \& Eysenck, 1975). O modelo big five inclui os fatores neuroticismo, extroversão, abertura à experiência, amabilidade e conscienciosidade. $\mathrm{O}$ fator neuroticismo alto, que tem sido relacionado com os estados de afeto negativo nos adultos, parece dominar o mau desempenho no IGT (Denburg et al., 2009), embora com resultados contraditórios (John \& Srivastava, 1999).O modelo dos três fatores de Eysenck (Eysenck \& Eysenck, 1975) inclui apenas as dimensões extroversão, neuroticismo e psicoticismo. O neuroticismo define a dimensão estabilidade/ansiedade; a extroversão define a dimensão da sociabilidade/introversão, amistosidade/ reserva, impulsividade/ reflexividade e aproximação do risco /evitamento do risco; e o psicoticismo define a dimensão do isolamento/aceitação social, insensibilidade aos outros/ preocupação com os outros. Com esse modelo, Hooper, Luciana, Wahlstrom, Conklin e Yarger (2008) verificaram, também, que, nos adolescentes saudáveis, o neuroticismo se associa negativamente ao desempenho no IGT. Nesse estudo, a medida de externalização, reportada pelos pais (Achenbach, 1991), evidenciou interagir com o neuroticismo e a extroversão, predizendo o desempenho no IGT.

No entanto, esses resultados podem ser influenciados pela inteligência cognitiva, mais que a emocional (Webb, DelDonno, \& Killgore, 2014), e pela idade em que é realizada a avaliação (Icellioglu \& Ozden, 2012). Nesse caso, por exemplo, a adolescência e o envelhecimento são períodos marcados por comportamentos de risco e de externalização diferenciados (Hooper et al., 2008; Nguyen et al., 2013), sendo estes condicionados por variáveis socioeconômicas (Ursache, \& Raver, 2015). De qualquer modo, esses comportamentos de risco e externalização tendem a associar-se a elevados níveis de neuroticismo (Heaven, Newbury, \& Mak, 2004), constituindo, particularmente no sexo masculino, um preditor significativo do desempenho no IGT (Hooper et al., 2008), eventualmente devido ao impacto dos afetos negativos na tomada de decisão (Bagneux, Font, \& Bollon, 2013; Mardaga \& Hansenne, 2015). Outros estudos evidenciam isso mesmo ao fornecerem indicações complementares sobre o efeito desses afetos negativos associados ao neuroticismo nas funções executivas (Preston, Buchanan, Stansfield, \& Bechara, 2007).

Um outro modelo explicativo dos resultados no IGT tem sido desenvolvido com base na teoria da personalidade de Gray (1970), considerando a influência direta ou integrada do sistema de inibição comportamental (BIS) e do sistema de ativação comportamental (BAS) na tomada de decisão. $\mathrm{O}$ modelo pressupõe que o BIS é ativado em resposta a ameaças/riscos, levando a um aumento do comportamento de inibição/evitamento; e o BAS, em função das respostas aos estímulos de recompensa (Carver, 2004). Em geral, a teoria da personalidade de Gray prediz que os sujeitos com um alto BIS são mais sensíveis às punições, enquanto os indivíduos com alto BAS são mais sensíveis às recompensas. Alguns autores têm, assim, evidenciado que a combinação baixo BIS - alto BAS (típica do fearlessness psicopático, por exemplo) implica um desempenho desvantajoso no IGT, ao contrário do que acontece quando os sujeitos apresentam um perfil alto BIS - baixo BAS (Ba, Zhang, Peng, Salvendy, \& Crundall, 2016; van Honk, Hermans, Putman, Montagne, $\&$ Schutter, 2002). Tal acontece devido à distribuição dos reforços e das punições nos diferentes baralhos não ser 
ponderada da mesma forma pelos diferentes sujeitos. No caso dos estados emocionais negativos, domina o baixo BIS - alto BAS enquanto os estados positivos, ao contrário, tendem a associar-se a um tipo de reatividade BIS mais alto (i.e., aversão à perda aumentada), assumindo a frequência das perdas esperadas um papel fundamental (Nygren, 1998). No entanto, outros autores verificaram que o bom desempenho no IGT está positivamente relacionado com o BAS, em particular com a sensibilidade à recompensa, mas não com o BIS (Franken \& Muris, 2005). Esses últimos autores e, mais recentemente, Yang, Zhong, Wu, Li e Li (2016) concluem, também, que o IGT não está relacionado com a impulsividade, embora Gray (1970) tenha usado esse termo para caracterizar a sensibilidade ao alto BAS.

Não obstante, a impulsividade tem sido associada às decisões desvantajosas no IGT, porque dificulta a aprendizagem, torna os indivíduos mais sensíveis à recompensa e é um sintoma de outras perturbações associadas ao mau desempenho na tarefa (Cackowski et al., 2014; Davis, Patte, Tweed, \& Curtis, 2007; Kertzman, Kagan, Vainder, Lapidus, \& Weizman, 2013; Takano, Takahashi, Tanaka, \& Hironaka, 2010; Upton, Bishara, Ahn, \& Stout, 2011; Stevens et al., 2015). A investigação sobre a importância dessa variável no desempenho no IGT, no entanto, tem mostrado resultados inconsistentes (Davis et al., 2007; Le Bas, Hughes, \& Stout, 2015; Upton et al., 2011), particularmente em amostras não clínicas (Franken \& Muris, 2005; Goudriaan, Grekin, \& Sher, 2007; Penolazzi, Leone, \& Russo, 2013), podendo inclusive estar relacionada com os ciclos menstruais / balanços hormonais (Derntl, Pintzinger, Kryspin-Exner, \&, Schöpf, 2014, Evans \& Hampson, 2014; Iannello, Biassoni, Nelli, Zugno, \& Colombo, 2015) ou até perceções subjetivas de fome (de Ridder, Kroese, Adriaanse, \&Evers, 2014).

Essa inconsistência dos resultados, para além dos problemas metodológicos ligados ao não controle de variáveis confundentes, pode estar relacionada com a complexidade do próprio constructo, particularmente com a sua multidimensionalidade, o que implica diferentes metodologias de avaliação e diferentes manifestações neurocognitivas, com consequências comportamentais distintas (Sharma, Markon, \& Clark, 2014; Vassileva, Georgiev, Martin, Gonzalez, \& Segala, 2011). Nesse caso, por exemplo, a impulsividade cognitiva tem sido associada à incapacidade de decidir de acordo com objetivos definidos a longo termo, em vez de a curto prazo, tal como são avaliados pelo IGT ou por outras tarefas semelhantes. A impulsividade, nesse caso, refletiria certa "miopia" em relação às consequências futuras do comportamento, podendo também ser entendida através do desconto temporal das recompensas futuras, isto é, pela redução do valor atual de uma recompensa futura, em função do atraso imposto a essa recompensa.

Efetivamente, alguns estudos têm verificado que o mau desempenho no IGT está associado a essa dimensão da impulsividade (Crone, Vendel, \& van der Molen, 2003; Franken \& Muris, 2005), mas outros evidenciam que a impulsividade (como fenômeno cognitivo) pode ser, afinal, um fenômeno independente da sensibilidade à recompensa (Franken, Muris, \& Rassin, 2005) ou constituir uma variável complexa com resultados diferenciados em função de outras variáveis moderadoras, como a aprendizagem (Upton et al., 2011), os estados de humor negativos, a procura de sensações e o investimento na tarefa, particularmente no que diz respeito à seleção preferencial do baralho B (Buelow \& Suhr, 2013).

Outro tipo de impulsividade, ainda, tem sido evidenciado em sujeitos que se descrevem como cognitivamente reflexivos e deliberativos, mas que apresentam, em outras medidas, traços impulsivos, aqui designados, por essa razão, de implícitos (Takano et al., 2010). Nesse caso, o problema está diretamente ligado à natureza do constructo, colocando a questão da impulsividade poder ser caracterizada como um traço de personalidade, definindo um comportamento sistemático que estaria nos antípodas do que se designa por traço deliberativo. Isto é, uma pessoa dita não impulsiva pode acabar por sê-lo em certas situações, sendo o contrário também possível. De outro modo, o problema pode ser colocado pressupondo a existência de uma dissociação entre o pensamento racional consciente e a origem inconsciente, implícita, da tomada de decisão. Considerando esse pressuposto, Takano et al. (2010) verificaram que apesar do número total de seleções conjuntas dos baralhos A' e B' decrescer ao longo dos ensaios (como esperado), não se observou uma diminuição clara das seleções do baralho B' e alguns sujeitos persistiam em escolher esse baralho de risco (B') mesmo quando as perdas aumentavam. O fenômeno é descrito como um efeito do reforço paradoxal das decisões de alto risco, depois de terem sido sofridas repetidamente grandes perdas monetárias. Propõem, como hipótese explicativa, a possibilidade de os jogadores especularem sobre as características dos baralhos, acreditando (como referido por alguns desses sujeitos) que o baralho B' contem um "grande ganho", que compensará todas as perdas. Por outras palavras, o pensamento lógico e deliberativo pode levar alguns indivíduos a assumirem e a manterem decisões de grande risco, com consequências finais negativas, e a esconderem (ou justificar racionalmente) um movimento impulsivo não consciente. Efetivamente, um dos resultados mais surpreendentes desse estudo é o fato de os sujeitos avaliados como deliberativos/reflexivos terem perdas persistentes, resultantes de um comportamento que pode ser percebido como impulsivo. Assim, esses resultados sugerem que a diferença entre deliberação explícita e impulsividade implícita poderá estar na origem de alguns resultados surpreendentes obtidos com o baralho B'. Quer dizer, também aqui o constructo relativo ao traço de personalidade caracterizado como deliberativo/reflexivo deve ser repensado (Takano \& Tanno, 2009).

A associação da impulsividade com a variável procura de sensações foi estudada utilizando uma versão modificada da tarefa, o IGT-v (van den Bos, Houx, \& Spruijt, 2006). Nessa versão, a diferença na magnitude das recompensas imediatas entre baralhos desvantajosos e vantajosos é aumentada, mantendo fixo o ganho líquido. Com esse esquema, Penolazzi et al. (2013) verificaram que os indivíduos mais impulsivos e com maior tendência para a procura de sensações tendem a fazer escolhas desvantajosas, ocorrendo o oposto com os participantes altamente sensíveis à punição. Esses resultados deixam em aberto a possibilidade de haver uma ligação entre essas variáveis, mas Young, Gudjonsson, Goodwin, Derek e Robin (2013) obtiveram resultados diferentes, 
nomeadamente a independência das variáveis procura de sensações e impulsividade nos resultados do IGT. Contudo, Xu, Korczykowski, Zhu e Rao (2013) salientam que tal pode estar associado com o fato de a impulsividade constituir um construto complexo e não relacionado apenas com traços específicos. Efetivamente, os estudos que avaliam o traço procura de sensações são relativamente inconsistentes (Penolazzi et al., 2013), evidenciando associações negativas (Crone et al., 2003; Glicksohn \& Zilberman, 2010), positivas (Reavis \& Overman, 2001) ou nulas (Brand \& AltstötterGleich, 2008). Não é claro, no entanto, se esses resultados são uma consequência direta dessas variáveis ou influenciados por outras, como a dificuldade de aprendizagem das contingências de reforço no IGT, a procura efetiva do risco após identificação da natureza dos baralhos ou diferentes tipos de comportamento impulsivo (Takano \& Tanno, 2009), ou mesmo se essa inconsistência é apenas fruto da falta de validade convergente entre o IGT e uma das medidas mais comuns de impulsividade, o Barratt Impulsivity Scale 11 (Vasconcelos, Sergeant, Corrêa, Mattos, \& Malloy-Diniz, 2014).

Em síntese, constata-se a inconsistência dos constructos e dos métodos de avaliação, bem como a interação das múltiplas variáveis de personalidade, em particular a impulsividade e a procura de sensações. Esses fenômenos podem estar na base desses resultados contraditórios obtidos pela investigação com o IGT.

\section{IGT, Psicopatia e Abuso de Substâncias}

Apesar de o DSM-IV TR (American Psychiatric Association, 2000) apenas considerar o diagnóstico de perturbação de personalidade antissocial, a psicopatia tem sido estudada como uma entidade específica dessa perturbação (Dolan \& Fullam, 2006), agrupando populações com uma etiologia comum, marcadas pela disfunção do processamento emocional (Blair, Mitchell, \& Blair, 2005). Nesse âmbito, a psicopatia tem sido associada a um comportamento de tomada de decisão sob risco, estudado com o IGT (Bechara et al., 1994; Bechara \& Damasio, 2002), à semelhança do que acontece com os pacientes com lesões orbitofrontais (Blair, Colledge, \& Mitchell, 2001). Na realidade, a natureza comum aos dois fenômenos parece resultar do fato de esses pacientes desenvolverem formas psicopáticas adquiridas (Barrash, Tranel, \& Anderson, 2000), evidenciando ambos uma insistência nas escolhas desvantajosas no IGT, o que tem sido justificado com base na hipótese do marcador somático (Damásio, 1995).

No entanto, os estudos com o IGT nas populações psicopáticas, ou com traços psicopáticos, têm evidenciado resultados inconsistentes (van Honk et al., 2002). Alguns concluem que esses pacientes apresentam uma propensão para selecionarem os baralhos mais arriscados (Blair et al., 2001; Mahmut, Homewood, \& Stevenson, 2008; van Honk et al., 2002), particularmente quando pressionados (Carre \& Jones, 2016). Outros, contudo, não verificam essa tendência (Takahashi, Takagishi, Nishinaka, Makino, \& Fukui, 2014), constatando antes que é o nível de ansiedade, e não a psicopatia, que melhor prediz esse comportamento
(Wang, Krajbich, Adolphs, \& Tsuchiya, 2012), o que aliás está de acordo com a evidência da relação entre a ansiedade traço e a aversão ao risco (Lorian, Mahoney, \& Grisham, 2012). Esses resultados, no entanto, são contraditórios com os obtidos por Zhang, Wang, Zhu, Yu e Chen (2015), em que o fenômeno da ansiedade traço parece ter uma distribuição não linear, com os grupos mais e menos ansiosos a obterem os piores desempenhos, ao contrário do grupo com valores médios de ansiedade traço.

Os resultados contraditórios obtidos com as populações psicopáticas, ou com traços psicopáticos, podem estar relacionados com dificuldades específicas ao constructo, particularmente com a estrutura bifatorial da check list mais utilizada na investigação na área, a Psychopathy Checklist-Revised (PCL-R; Hare, 1991). Isto é, com a dimensão primária (Fator 1 [F1]) e a dimensão secundária (Fator 2 [F2]): a primeira (F1), refletindo frieza afetiva, ausência de culpa, charme superficial, falta de empatia e agressividade instrumental, e a segunda (F2), comportamentos antissociais, impulsividade, falta de controle e agressividade predominantemente reativa (Ferrigan, Valentiner, \& Berman, 2000). Essas duas dimensões podem estar associadas a entidades clínicas distintas e podem apresentar condições divergentes no que respeita à ativação do sistema nervoso autônomo (Patrick, Bradley, \& Lang, 1993), às capacidades cognitivas (Ross, Benning, \& Adams, 2007), ao abuso de substâncias (Taylor \& Lang, 2006) e ao comportamento antissocial (Walters, 2003). Nesse sentido, alguns autores têm verificado que só a psicopatia secundária (F2) se relaciona com o esperado desempenho de risco no IGT (Dean et al., 2013; Wang et al., 2012).

De qualquer modo, a psicopatia surge frequentemente associada a uma alta comorbidade com perturbações do Eixo I e do Eixo II da DSM-IV TR (American Psychological Association, 2000), nomeadamente o abuso de substâncias (Vassileva et al., 2011) e as perturbações de personalidade borderline e antissocial (He, Cassaday, Howard, Khalifa, \& Bonardi, 2011). No caso da associação frequente entre abuso de substâncias e psicopatia, por exemplo, as evidências de comportamentos de risco na tomada de decisão avaliada pelo IGT são grandes (Vassileva et al., 2011; Vassileva, Gonzalez, Bechara, \& Martin, 2007), mas a importância decisiva dos comportamentos antissociais nesses resultados mantém-se. Outros estudos têm, também, relacionado esses comportamentos com o abuso de substâncias (Brinkley, Schmitt, Smith, \& Newman, 2001). Nesses casos, os problemas de comportamento e o desvio antissocial seriam os verdadeiros responsáveis pelo comportamento de risco observado no IGT, ao contrário da questão emocional, relacionada com um melhor funcionamento das funções executivas, o que se traduziria no sucesso de muitos desses sujeitos no mundo financeiro e político (Levenson, 1992). Portanto, parte da variabilidade nos resultados pode estar associada às dimensões mais adaptativas da psicopatia não criminal (Zimak, Suhr, \& Bolinger, 2014), incluindo a inteligência criativa e o pensamento divergente apresentados por alguns desses sujeitos (Galang, Castelo, Santos, Perlas, \& Angeles, in press). No entanto, essas últimas observações devem ser tomadas com alguma precaução, dada a 
especificidade dos procedimentos de diagnóstico assumidos nesse estudo.

De qualquer modo, os traços psicopáticos associados ao desvio antissocial podem ajudar a explicar os resultados inconsistentes no IGT. Miranda, MacKillop, Meyerson, Justus e Lovallo (2009), por exemplo, verificaram que os alcoólicos com um diagnóstico de personalidade antissocial fazem seleções mais desvantajosas no IGT do que os alcoólicos sem personalidade antissocial. No entanto, verificaram também que o comportamento antissocial se associava ao F2 do Psychopathic Personality Inventory (Lilienfeld \& Andrews, 1996) quando ocorriam mais comportamentos de risco no IGT. Fenômeno semelhante foi observado por Lösel e Schmucker (2004), usando o PCL-R (Hare, 1991). Nesse caso, apenas F2 se encontrava positivamente correlacionado com as escolhas de risco, enquanto F1 se apresentava negativamente correlacionado com essas seleções, embora nenhuma das correlações se apresentasse estatisticamente significativa. Do mesmo modo, o fato de F2 assumir a principal responsabilidade pelas escolhas desvantajosas observadas explica como a impulsividade (associada à psicopatia e aos comportamentos antissociais) pode justificar a incapacidade desses sujeitos diferirem a gratificação e aprenderem com a experiência (Dean et al., 2013). No entanto, a maioria das medidas de psicopatia secundária colocam questões que simulam um tipo impulsivo (e.g., Lilienfeld \& Andrews, 1996), o que poderá também querer dizer que essa variável deve ser relacionada com o desempenho no IGT, preferencialmente em combinação com outros traços da personalidade, nomeadamente os outros traços psicopáticos associados a F2.

Por outro lado, parte da variabilidade dos resultados obtidos com essas populações pode estar associada a diferenças amostrais, à ausência de procedimentos de controle de variáveis confundentes (ansiedade em particular), mas também ao fato de os estudos não terem considerado as diferentes dimensões do construto e respectivas diferenciações de traços psicopáticos. Nesse caso, incluímos a estrutura de quatro dimensões (Hare \& Neumann, 2008), de quatro fatores (Hare, 2003) e a estrutura de três fatores hierárquicos (com F1 - interpessoal, F2 - afetivo e F3 impulsivo; Cooke \& Michie, 1997). Essa estrutura exclui os itens mais ligados ao comportamento antissocial e assume uma posição clara na controvérsia relativa à conjugação do fenômeno antissocial no construto psicopático. Nesse caso, os comportamentos antissociais são considerados não necessários à definição de traço de personalidade psicopático (Filho, Teixeira, \& Martins de Almeida, 2014). Contudo, de acordo com o nosso conhecimento, essa estrutura não foi, até ao momento, explorada com o IGT.

Considerando especificamente a estrutura de quatro dimensões (1.a - interpessoal; 1.b - afeto; 2.a - estilo de vida/impulsividade; e 2.b - antissocial), Hughe, Dolan, Trueblood e Stout (2015) exploraram a associação de cada uma dessas dimensões com o IGT. Os resultados são, no entanto, paradoxais com o que temos vindo a referir, já que a faceta 2.b (que não inclui os itens relativos ao estilo de vida/impulsividade) se relaciona com uma melhor performance no IGT, embora observem também que esses psicopatas criminosos, no geral, não obtêm resultados no
IGT significativamente diferentes dos verificados no grupo de controle.

De qualquer modo, a investigação tem revelado que os dois tipos de psicopatia (criminosa e não criminosa) apresentam grandes semelhanças (Vanman, Mejia, Dawson, Schell, \& Raine, 2003), particularmente no que concerne aos processos empáticos (Benning, Patrick, \& Iacono, 2005; Gao\& Raine, 2010). A relação etiológica e genotípica entre esses dois fenômenos, no entanto, não está validada, podendo os constructos não serem os mesmos (Mahmut et al., 2008) e a interferência de outras variáveis ter implicações diferentes em um e em outro caso (van Honk et al., 2004).

A associação entre adição e psicopatia é outra forma de tentar esclarecer a transformação qualitativa do fenômeno quando há interceção de variáveis. Segundo Vassileva et al. (2007), verifica-se que aproximadamente $25 \%$ dos dependentes de substâncias apresentam níveis elevados de psicopatia, chegando aos 37,5\% nos dependentes de heroína. Essa associação pode resultar em um efeito cumulativo em detrimento da capacidade de tomada de decisão. Efetivamente, os estudos que se focam apenas no abuso de substâncias evidenciam uma tendência de enviesamento motivacional, no sentido de esses sujeitos preferirem os ganhos imediatos (consistente com a alta sensibilidade à recompensa) aos ganhos diferidos (mesmo que maiores).

Apesar de os dependentes de substâncias evidenciarem também, tendencialmente, mau desempenho no IGT (e.g., Bechara \& Damasio, 2002; Palomo, Beninger, Kostrzewa, \& Archer, 2008), alguns estudos concluem que esses resultados estão mais ligados à interceção de outras variáveis, como as características de personalidade, incluindo a psicopatia (Taylor \& Lang, 2006) e a impulsividade, no caso do alcoolismo (Körner, Schmidt, Soyka, 2015), mais do que ao efeito direto da intoxicação (Balodis, Macdonald, \& Olmstead, 2006). Provavelmente, por essa razão, os estudos com dependentes de substâncias evidenciam uma grande variabilidade nos resultados (e.g., Bechara \& Damasio, 2002; Bechara et al., 2001; Bechara \& Martin, 2004). Nesse caso, as dificuldades podem ser relativas às adaptações neuroquímicas e neurocerebrais associadas ao abuso de drogas, particularmente à ativação diferenciada da região préfrontal dorsolateral direita e do striatum ventral; a primeira associando-se a maiores comportamentos de risco e a segunda a um maior evitamento desses comportamentos (Yamamoto, Woo, Wager, Regner, \& Tanabe, 2015), ou mesmo a ativações específicas associadas ao transporte da serotonina (5-HT) em alcoólicos (Lovallo et al., 2014); aos fatores de risco causais, anteriores ao consumo das substâncias (Davis et al., 2007), ou, ainda, à forte comorbilidade associada a esses comportamentos.

Assim, por exemplo, considerando a influência da antissociabilidade e da extensão do policonsumo de drogas na impulsividade cognitiva e motora entre dependentes de substâncias, primariamente de cocaína ou heroína, Vassileva et al. (2007) verificaram que, ao contrário do esperado, a antissociabilidade nesses sujeitos estava associada ao desempenho mais vantajoso no IGT, independentemente da extensão do abuso de drogas. Os resultados sugerem uma distribuição não linear, em que as formas subclínicas de antissociabilidade podem ter um efeito facilitador paradoxal 
na tomada de decisão entre dependentes de substâncias, ao contrário do que pode acontecer nas formas clínicas.

Os dependentes de opiáceos evidenciam dificuldades no controle dos impulsos, incluindo tomada de decisão impulsiva, déficts na inibição comportamental, comportamentos de risco e um maior desconto temporal das recompensas futuras, mas esses resultados são difíceis de interpretar, dada a dificuldade de os dissociar das outras variáveis confundentes (Hulka et al., 2015; Vassileva et al., 2011).

De qualquer modo, o efeito potencialmente confundente da comorbidade, que atinge intensamente o abuso de substâncias, pode ser considerado olhando para os diferentes estudos que relacionam déficts no desempenho com o IGT e outras perturbações, tais como as perturbações borderline da personalidade (Haaland \& Landro, 2007; LeGris et al., 2012; LeGris, Toplak, \& Links, 2014) ou mesmo, simplesmente, a afetação específica da memória de trabalho causada pelo alcoolismo crônico (Brevers et al., 2014).

Outro tipo de evidências, ligadas aos comportamentos de risco com condutores rodoviários, por exemplo, suportam a ideia de que os resultados no IGT estão essencialmente ligados a um conjunto complexo de variáveis organizadas em perfis cognitivos e de personalidade. Nesse caso, as variáveis fearlessness (psicopatia), descontrole/desinibição comportamental (alcoolismo), neuroticismo (traço de personalidade), ansiedade e diminuição das respostas das hormonas corticosteroides ao estresse (cortisol) (Brown et al., 2016), ou mesmo, como referido, a afetação específica da memória de trabalho (Brevers et al., 2014), interatuam configurando tipos de resposta decisional.

Finalmente, Ursache e Raver (2015) evidenciam outra realidade, isto é, o IGT tende a apresentar resultados desvantajosos com grande significância estatística (incluindo a predominância do baralho B) quando os grupos incluem sujeitos de nível socioeconômico muito baixo. Esses sujeitos evidenciam um desconto temporal das recompensas futuras idêntico ao evidenciado pelos lesionados préfrontais ventromediais (Bechara et al., 1994).Um fenômeno semelhante, em sujeitos colocados em um outro extremo socioeconômico, foi detectado por Mussel, Reiter, Osinsky e Hewig (2015) ao verificarem o "valor" da "ganância" nesse desconto temporal, com consequências muitas vezes catastróficas para o mundo empresarial e financeiro em que ocorrem. Quer dizer, em síntese, a decisão desvantajosa medida pelo IGT pode estar associada a experiências de pobreza material e de sobrevivência implícita a essa experiência (na medida em que essa sobrevivência é incompatível com o adiamento da recompensa) ou ao alto valor subjetivo da recompensa e do risco em populações no outro extremo dessa distribuição. Esses dois extremos evidenciam uma outra não linearidade do fenômeno, semelhante à já observada por Zhang et al. (2015) para a ansiedade.

\section{Considerações Finais}

A pesquisa na área tem evidenciado um conjunto de indicações, das quais destacamos as seguintes:
1. Considerando os modelos de personalidade big five e o modelo dos três fatores de Eysenck, o neuroticismo surge, em muitos estudos, associado negativamente ao desempenho no IGT;

2. Essa relação pode, no entanto, estar condicionada pelo efeito dos afetos negativos associados ao neuroticismo nas funções executivas;

3. Essa indicação é complementada pelos estudos com o modelo de Gray (1970), segundo os quais a combinação baixo BIS - alto BAS, que caracteriza não apenas a psicopatia mas também os estados emocionais negativos, evidencia um desempenho desvantajoso no IGT;

4. A variável impulsividade, de modo geral, tem sido associada a maus resultados no IGT, mas a pesquisa tem mostrado resultados inconsistentes;

5. Esses resultados inconsistentes podem estar relacionados com a complexidade do próprio constructo, particularmente com a sua multidimensionalidade e, inclusive, com as diferentes metodologias utilizadas para o avaliar, mas quase sempre reportadas a um mesmo fenômeno;

6. Também os estudos que avaliam o traço procura de sensações evidenciam resultados contraditórios;

7. Na psicopatia, as escolhas desvantajosas no IGT, parecem estar mais relacionadas com F2(fator associado à antissociabilidade no PCL-R de Hare, 1991) e menos a F1 do mesmo construto (psicopatia primária). Esse resultado vai ao encontro de uma literatura crescente, sugerindo que a psicopatia secundária é um melhor preditor dos problemas de tomada de decisão que a psicopatia primária;

8. Contudo, a exploração das diferentes estruturas do PCL-R, considerando particularmente as estruturas de três e quatro fatores, é praticamente inexistente, pelo que muitos desses resultados podem ser específicos a dimensões/fatores não diferenciados na estrutura de dois fatores;

9. Considerando apenas essa estrutura de dois fatores, os resultados com os dependentes de substâncias (particularmente heroinômanos) parecem estar relacionados com a antissociabilidade e com a variável descontrole/desinibição comportamental;

10. Contudo, o efeito potencialmente confundente e não linear das múltiplas variáveis (ansiedade, comorbidade psicopatológica, nível socioeconômico e valor subjetivo atribuído ao ganho) podem justificar parte da inconsistência dos resultados.

Em síntese, parte dessa inconsistência pode estar relacionada não apenas com os constructos teóricos subjacentes a muitas das variáveis em estudo (a impulsividade é, neste caso, o exemplo mais claro), mas também com os métodos de avaliação propostos e utilizados para medir essas variáveis. Efetivamente, em muitas situações, a validade de critério conceptual e facial desses métodos não está devidamente esclarecida, pelo que muitos dos resultados podem estar relacionados, simplesmente, com construtos diferentes. Ainda, os estudos com populações clínicas são sistematicamente enviesados pela presunção de linearidade das distribuições e pela dificuldade em controlar devidamente 
esses efeitos. Essas dificuldades e limitações são, na nossa perspetiva, indicações fundamentais para o futuro da pesquisa na área.

\section{Referências}

Achenbach, T. M. (Ed.). (1991). Integrative guide for the 1991 CBCL/4-18, YSR and TRF profiles. Burlington: Department of Psychiatry, University of Vermont.

American Psychiatric Association. (2000). Diagnostic and statistical manual of mental disorders (4 ed.). Washington, DC: American Psychiatric Association.

Ba, Y., Zhang, W., Peng, Q., Salvendy, G., \& Crundall, D. (2016). Risk-taking on the road and in the mind: Behavioural and neural patterns of decision making between risky and safe drivers. Ergonomics,59(1), 27-38. doi: 10.1080/00140139.2015.1056236

Bagneux, V., Font, H., \& Bollon, T. (2013). Incidental emotions associated with uncertainty appraisals impair decisions in the Iowa Gambling Task. Motivation and Emotion, 37(4), 818-827. doi: 10.1007/s11031-013-9346-5

Balodis, I. M., MacDonald, T. K., \& Olmstead, M. C. (2006). Instructional cues modify performance on the Iowa Gambling Task. Brain and Cognition, 60(2), 109-117. doi:10.1016/j. bandc.2005.05.007

Barrash, J., Tranel, D., \& Anderson, S. W. (2000). Acquired personality disturbances asssociated with bilateral damage to the ventromedial prefrontal region. Developmental Neuropsychology, 18(3), 355-381. doi:10.1207/ S1532694205Barrash

Bechara, A., Damasio, A. R., Damasio, H., \& Anderson, S. W. (1994). Insensitivity to future consequences following damage to human prefrontal cortex. Cognition, 50(1-3), 7-15. doi:10.1016/0010-0277(94)90018-3

Bechara, A., \& Damasio, H. (2002). Decision-making and addiction (part I): Impaired activation of somatic states in substance dependent individuals when pondering decisions with negative future consequences. Neuropsychologia, 40(10), 1675-1689. doi:10.1016/S0028-3932(02)00015-5

Bechara, A., Dolan, S., Denburg, N., Hindes, A., Anderson, S. W., \& Nathan, P. E. (2001). Decision-making deficits, linked to a dysfunctional ventromedial prefrontal cortex, revealed in alcohol and stimulant abusers. Neuropsychologia, 39(4), 376389. doi:10.1016/S0028-3932(00)00136-6

Bechara, A., \& Martin, E. M. (2004). Impaired decision making related to working memory deficits in individuals with substance addictions. Neuropsychology, 18(1), 152-162. doi:10.1037/0894-4105.18.1.152

Benning, S. D., Patrick, C. J., \& Iacono, W. G. (2005). Psychopathy, startle blink modulation, and electrodermal reactivity in twin men. Psychophysiology, 42(6), 753-762. doi:10.1111/j.14698986.2005.00353.x

Blair, R. J. R., Colledge, E., \& Mitchell, D. (2001). Somatic markers and response reversal: Is there orbitofrontal cortex dysfunction in boys with psychopathic tendencies? Journal of Abnormal Child Psychology, 29(6), 499-511. doi:10.1023/A:1012277125119

Blair, R. J. R., Mitchell, D., \& Blair, K. (2005). The psychopath: Emotion and the brain. Oxford, UK: Blackwell.
Buelow, M. T., \& Suhr, J. A. (2013). Personality characteristics and state mood influence individual deck selections on the Iowa Gambling Task. Personality and Individual Differences, 54(5), 593-597. doi:10.1016/j.paid.2012.11.019

Brand, M., \& Altstötter-Gleich, C. (2008). Personality and decision-making in laboratory gambling tasks - Evidence for a relationship between deciding advantageously under risk conditions and perfectionism. Personality and Individual Differences, 45(3), 226-231. doi:10.1016/j.paid.2008.04.003

Brevers, D.,Bechara, A., Cleeremans, A., Kornreich, C.,Verbanck, P., \& Noël, X. (2014). Impaired decision-making under risk in individuals with alcohol dependence. Alcoholism-Clinical and Experimental Research, 38(7), 1924-1931.doi:10.1111/ acer. 12447

Brinkley, C. A., Schmitt, W. A., Smith, S. S., \& Newman, J. P. (2001). Construct validation of a self-report psychopathy scale: Does Levenson's self-report psychopathy scale measure the same constructs as Hare's psychopathy checklist-revised? Personality and Individual Differences, 31(7), 1021-1038. doi:10.1016/S0191-8869(00)00178-1

Brown, T. G., Ouimet, M. C., Eldeb, M., Tremblay, J., Vingilis, E., Nadeau, L., Pruessner, J., \& Bechara, A. (2016). Personality, executive control, and neurobiological characteristics associated with different forms of risky driving. PLoS One,11(2), e0150227. doi:10.1371/journal.pone.0150227

Cackowski, S.,Reitz, A,C., Ende, G., Kleindienst, N., Bohus, M.,Schmahl, C.,\& Krause-Utz, A. (2014). Impact of stress on different components of impulsivity in borderline personality disorder. Psychological Medicine, 44(15), 3329-3340. doi:10.1017/S0033291714000427

Carre, J. R., \& Jones, D. N. (2016). The impact of social support and coercion salience on Dark Triad decision making. Personality and Individual Differences, 94, 92-95. doi:10.1016/j. paid.2016.01.006

Carver, C. S. (2004). Negative affects deriving from the behavioral approach system. Emotion, 4(1), 3-22. doi:10.1037/15283542.4.1.3

Cooke, D. J., \& Michie, C. (1997). An item response theory evaluation of Hare's Psychopathy Checklist. Psychological Assessment, 9(1), 2- 13. doi:10.1037/1040-3590.9.1.3

Costa, P. T., \& McCrae, R. R. (Eds.). (1992). Revised NEO Personality Inventory (NEO-PI-R) and NEO Five-Factor Inventory (NEO-FFI) manual. Odessa, FL: Psychological Assessment Resources.

Crone, E. A., Vendel, I., \& van der Molen, M. W. (2003). Decisionmaking in disinhibited adolescents and adults: Insensitivity to future consequences or driven by immediate reward? Personality and Individual Differences, 35(7), 1625-1641. doi:10.1016/s0191-8869(02)00386-0

Damásio, A. R. (1995). O erro de Descartes. Emoção, razão e cérebro humano (D. Vicente \& G. Segurado, Trans.). Mem Martins: Publicações Europa-América.

Davis, C., Patte, K., Tweed, S., \& Curtis, C. (2007). Personality traits associated with decision-making deficits. Personality and Individual Differences, 42(2), 279-290. doi:10.1016/j. paid.2006.07.006 
Dean, A. C., Altstein, L. L., Berman, M. E., Constans, J. I., Sugar, C. A., \& McCloskey, M. S. (2013). Secondary psychopathy, but not primary psychopathy, is associated with risky decisionmaking in noninstitutionalized young adults. Personality and Individual Differences, 54(2), 272-277. doi:10.1016/j. paid.2012.09.009

Denburg, N., Weller, J., Yamada, T., Shivapour, D., Kaup, A., LaLoggia, A., . . Bechara, A. (2009). Poor decision making among older adults is related to elevated levels of neuroticism. Annals of Behavioral Medicine, 37(2), 164-172. doi:10.1007/ s12160-009-9094-7

de Ridder, D., Kroese, F., Adriaanse, M., \& Evers, C. (2014). Always gamble on an empty stomach: Hunger is associated with advantageous decision making. PLoS One, 9(10), e111081. doi:10.1371/journal.pone.0111081

Derntl, B., Pintzinger, N., Kryspin-Exner, I., \& Schöpf , V. (2014). The impact of sex hormone concentrations on decision-making in females and males. Frontiers in Neuroscience, 8(352), 1-11. doi:10.3389/fnins.2014.00352

Dolan, M., \& Fullam, R. (2006). Face affect recognition deficits in personality disordered offenders: Association with psychopathy. Psychological Medicine, 36, 1563-1569. doi:10.1017/S0033291706008634

Evans, K. L., \& Hampson, E. (2014). Does risk-taking mediate the relationship between testosterone and decision-making on the Iowa Gambling Task? Personality and Individual Differences, 61(62), 57-62. doi:10.1016/j.paid.2014.01.011

Eysenck, H., \& Eysenck, S. (1975). Manual of the Eysenck Personality Inventory. San Diego: Educational and Industrial Testing Service.

Ferrigan, M. M., Valentiner, D. P., \& Berman, M. E. (2000). Psychopathy dimensions and awareness of negative and positive consequences of aggressive behaviour in a nonforensic sample. Personality and Individual Differences, 28(3), 527538. doi:10.1016/S0191-8869(99)00118-X

Filho, N. H., Teixeira, M. A., \& Martins de Almeida, R. M. (2014). Estrutura fatorial da escala Psychopathy Checklist-Revised (PCL-R): Uma revisão sistemática. Avaliação Psicológica, 13(2), 247-256. Retirado de http://pepsic.bvsalud.org/scielo. php?script $=$ sci_arttext\&pid=S1677-04712014000200012\&ln $\mathrm{g}=\mathrm{pt} \& \operatorname{tlng}=\mathrm{pt}$.

Franken, I. A., Muris, P., \& Rassin, E. (2005). Psychometric properties of the Dutch BIS/BAS Scales. Journal of Psychopathology and Behavioral Assessment, 27(1), 25-30. doi:10.1007/s10862-005-3262-2

Franken, I. H. A., \& Muris, P. (2005). Individual differences in decision-making. Personality and Individual Differences, 39(5), 991-998. doi:10.1016/j.paid.2005.04.004

Galang, A. J., Castelo, V. L., Santos III, L. C., Perlas, C. M., \& Angeles, M. A. (in press). Investigating the prosocial psychopath model of the creative personality: Evidence from traits and psychophysiology. Personality and Individual Differences. doi:10.1016/j.paid.2016.03.081

Gao, Y., \& Raine, A. (2010). Successful and unsuccessful psychopaths: A neurobiological model. Behavioural Sciences and the Law, 28, 194-210. doi:10.1002/bsl.924

Glicksohn, J., \& Zilberman, N. (2010). Gambling on individual differences in decision making. Personality and Individual Differences, 48(5), 557-562. doi:10.1016/j.paid.2009.12.006
Goudriaan, A. E., Grekin, E. R., \& Sher, K. J. (2007). Decision making and binge drinking: A longitudinal study. Alcoholism: Clinical \& Experimental Research, 31(6), 928-938. doi:10.1111/ j.1530-0277.2007.00378.x

Gray, J. A. (1970). The psychophysiological basis of introversionextraversion. Behaviour Research and Therapy, 8(3), 249-266. doi:10.1016/0005-7967(70)90069-0

Haaland, V. O., \& Landro, N. I. (2007). Decision making as measured with the Iowa Gambling Task in patients with borderline personality disorder. Journal of the International Neuropsychological Society, 13(4), 699-703. doi:10.1017/ S1355617707070890

Hare, R. D. (1991). The Hare Psychopathy Checklist - Revised. Toronto, ON: Multi-Health Systems.

Hare, R. D. (2003). Manual for the Revised Psychopathy Checklist (2nd ed.).Toronto, ON, Canada: Multi-Health Systems.

Hare, R. D., \& Neumann, C. S. (2008). Psychopathy as a clinical and empirical construct. Annual Review of Clinical Psychology, 4, 217246. doi:10.1146/annurev.clinpsy.3.022806.091452

He, Z., Cassaday, H., Howard, R., Khalifa, N., \& Bonardi, C. (2011). Impaired Pavlovian conditioned inhibition in offenders with personality disorders. Quarterly Journal of Experimental Psychology, 64(12), 2334-2351. doi:10.1080/17470218.2011 .616933

Heaven, P. C. L., Newbury, K., \& Mak, A. (2004). The impact of adolescent and parental characteristics on adolescent levels of delinquency and depression. Personality and Individual Differences, 36(1), 173-185. doi:10.1016/S01918869(03)00077-1

Hooper, C. J., Luciana, M., Wahlstrom, D., Conklin, H. M., \& Yarger, R. S. (2008). Personality correlates of Iowa Gambling Task performance in healthy adolescents. Personality and Individual Differences, 44(3), 598-609. doi:10.1016/j. paid.2007.09.021

Hughe, M. A., Dolan, M. C., Trueblood, J. S., \& Stout, J. C. (2015). Psychopathic personality traits and Iowa Gambling Task performance in incarcerated offenders. Psychiatry, Psychology and Law, 22(1), 134-144. doi:10.1080/13218719.2014.919689

Hulka, L. M.,Vonmoos, M., Preller, K. H., Baumgartner, M. R., Seifritz, E., Gamma, A., \& Quednow, B. B. (2015). Changes in cocaine consumption are associated with fluctuations in self-reported impulsivity and gambling decision-making. Psychological Medicine, 45(14), 3097-3110. doi:10.1017/ S0033291715001063

Iannello, P., Biassoni, F., Nelli, B., Zugno, E., \& Colombo, B. (2015). The influence of menstrual cycle and impulsivity on risk-taking behavior. Neuropsychological Trends, 17,47-52. doi:10.7358/neur-2015-017-iann

Icellioglu, S., \& Ozden, M. S. (2012). Personality effect on decisionmaking among normal university students and comparing Gambling Task performances of normal university students with a group of normal adults. Procedia - Social and Behavioral Sciences, 46(0), 1010-1014. doi:10.1016/j.sbspro.2012.05.240

John, O., \& Srivastava, S. (1999). The big five trait taxonomy: History, measurement, and theoretical perspectives. In L. A. Pervin \& O. P. John (Eds.), Handbook of personality: Theory and research (2 ed., pp. 102-138). New York: Guilford. 
Kertzman, S., Kagan, A.,Vainder, M., Lapidus, R., \& Weizman, A. (2013). Interactions between risky decisions, impulsiveness and smoking in young tattooed women. $B M C$ Psychiatry, 13(278),1-12 doi:10.1186/1471-244X-13-278

Körner, N., Schmidt, P., \& Soyka, M. (2015). Decision making and impulsiveness in abstinentalcohol-dependent people and healthy individuals: A neuropsychological examination. Substance Abuse Treament, Prevency and Policy, 10(24), 1-10 doi:10.1186/s13011-015-0020-7

Le Bas, G. A., Hughes, M. A., \& Stout, J. C. (2015). Utility of selfreport and performance-based measures of risk for predicting driving behavior in young people. Personality and Individual Differences, 86, 184-188. doi:10.1016/j.paid.2015.05.034

LeGris, J., Links, P. S., van Reekum, R., Tannock, R., \& Toplak, M. (2012). Executive function and suicidal risk in women with borderline personality disorder. Psychiatry Research, 196(1), 101-108. doi:10.1016/j.psychres.2011.10.008

LeGris, J. R. N., Toplak, M., C., \& Links, P. S. (2014). Affective decision making in women with borderline personality disorder. Journal of Personality Disorders, 28(5), 698-719. doi:10.1521/pedi_2014_28_140

Levenson, M. R. (1992). Rethinking psychopathy. Theory \& Psychology, 2(1), 51-71. doi:10.1177/0959354392021003

Lilienfeld, S. O., \& Andrews, B. P. (1996). Development and preliminary validation of a self-report measure of psychopathic personality traits in noncriminal population. Journal of Personality Assessment, 66(3), 488-524. doi:10.1207/ s15327752jpa6603 3

Lorian, C. N., Mahoney, A., \& Grisham, J. R. (2012). Playing it safe: An examination of risk-avoidance in an anxious treatmentseeking sample. Journal of Affective Disorders, 141(1), 63-71. doi:10.1016/j.jad.2012.02.021

Lösel, F., \& Schmucker, M. (2004). Psychopathy, risk taking, and attention: A differentiated test of the somatic marker hypothesis. Journal of Abnormal Psychology, 113(4), 522-529. doi:10.1037/0021-843X.113.4.522

Lovallo, W. R., Enoch, M. A., Yechiam, E., Glahn, D. C., Acheson, A., Sorocco, K. H.,... Goldman, D. (2014). Differential impact of serotonin transporter activity on temperament and behavior in persons with a family history of alcoholism in the Oklahoma family health patterns project. Alcoholism-Clinical and Experimental Research, 38(6), 1575-1581. doi:10.1111/ acer. 12412

Mahmut, M. K., Homewood, J., \& Stevenson, R. J. (2008). The characteristics of non-criminals with high psychopathy traits: Are they similar to criminal psychopaths? Journal of Research in Personality, 42(3), 679-692. doi:10.1016/j.jrp.2007.09.002

Mardaga, S., \& Hansenne, M. (2015). Personality and skin conductance responses to reward and punishment. Influence on the Iowa Gambling Task performance. Journal of Individual Differences, 33, 17-23.doi:10.1027/1614-0001/a000057

Miranda Jr, R., MacKillop, J., Meyerson, L. A., Justus, A., \& Lovallo, W. R. (2009). Influence of antisocial and psychopathic traits on decision-making biases in alcoholics. Alcoholism: Clinical and Experimental Research, 33(5), 817-825. doi:10.1111/j.1530-0277.2009.00901.x

Mussel, P., Reiter, A. M., Osinsky, R., \& Hewig, J. (2015). State- and trait-greed, its impact on risky decision-making and underlying neural mechanisms. Social Neuroscience, 10(2), 126-134. do i: $10.1080 / 17470919.2014 .965340$
Nguyen, C. M., Barrash, J., Koenigs, A. L., Bechara A.,Tranel D.,Denburg, N. L. (2013). Decision-making deficits in normal elderly persons associated with executive personality disturbances. International Psychogeriatrics, 25(11), 18111819.doi:10.1017/S1041610213001270

Nygren, T. E. (1998). Reacting to perceived high- and low-risk win-lose opportunities in a risky decision-making task: Is it framing or affect or both? Motivation and Emotion, 22(1), 73-98. doi:10.1023/a:1023096709380

Palomo, T., Beninger, R., Kostrzewa, R., \& Archer, T. (2008). Focusing on symptoms rather than diagnoses in brain dysfunction: Conscious and nonconscious expression in impulsiveness and decision-making. Neurotoxicity Research, 14(1), 1-20. doi:10.1007/bf03033572

Patrick, C. J., Bradley, M. M., \& Lang, P. J. (1993). Emotion in the criminal psychopath: Startle reflex modulation. Journal of Abnormal Psychology, 102(1), 82-92. doi:10.1037/0021843X.102.1.82

Penolazzi, B., Leone, L., \& Russo, P. M. (2013). Individual differences and decision making: When the lure effect of gain is a matter of size. PLoS One, 8(3), e58946. doi:10.1371/ journal.pone.0058946

Preston, S. D., Buchanan, T. W., Stansfield, R. B., \& Bechara, A. (2007). Effects of anticipatory stress on decision making in a gambling task. Behavioral Neuroscience, 121(2), 257-263. doi:10.1037/0735-7044.121.2.257

Reavis, R., \& Overman, W. (2001). Adult sex differences on a decision-making task previously shown to depend on the orbital prefrontal cortex. Behavioral Neuroscience, 115(1), 196-206. doi:10.1037/0735-7044.115.1.196

Ross, S., Benning, S., \& Adams, Z. (2007). Symptoms of executive dysfunction are endemic to secondary psychopathy: An examination in criminal offenders and noninstitutionalized young adults. Journal of Personality Disorders 21(4), 384-399. doi:10.1521/pedi.2007.21.4.384

Sharma, L., Markon, K. E., \& Clark, L. A. (2014). Toward a theory of distinct types of "impulsive" behaviors: A meta-analysis of self-report and behavioral measures. Psychological Bulletin, 140(2),374-408. doi:10.1037/a0034418

Steingroever, H., Wetzels, R., Horstmann, A., Neumann, J., \& Wagenmakers, E.-J. (2013). Performance of healthy participants on the Iowa Gambling Task. Psychological Assessment, 25(1), 180-193. doi:10.1037/a0029929

Stevens, L.,Goudriaan, A. E.,Verdejo-Garcia, A.,Dom, G., Roeyers, H., \& Vanderplasschen, W. (2015). Impulsive choice predicts short-term relapse in substance-dependent individuals attending an in-patient detoxification programme. Psychological Medicine, 45(10), 2083-2093. doi:10.1017/ S003329171500001X

Takahashi, T.,Takagishi, H.,Nishinaka, H., Makino, T., \&Fukui, H. (2014). Neuroeconomics of psychopathy: Risk taking in probability discounting of gain and loss predicts psychopathy. Neuroendocrinology Letters, 35(6), 510-517. Retirado de http://www.nel.edu/home.htm

Takano, K., \& Tanno, Y. (2009). Self-rumination, self-reflection, and depression: Self-rumination counteracts the adaptive effect of self-reflection. Behaviour Research and Therapy, 47(3), 260-264. doi:10.1016/j.brat.2008.12.008 
Takano, Y., Takahashi, N., Tanaka, D., \& Hironaka, N. (2010). Big losses lead to irrational decision-making in gambling situations: Relationship between deliberation and impulsivity. PLoS One, 5(2), 1-6. doi:10.1371/journal.pone.0009368

Taylor, J., \& Lang, A. (2006). Psychopathy and substance use disorders. In C. Patrick (Ed.), Handbook of psychopathy (pp. 495-511). Guilford Press: New York.

Upton, D. J., Bishara, A. J., Ahn, W. Y., \& Stout, J. C. (2011). Propensity for risk taking and trait impulsivity in the Iowa Gambling Task. Personality and Individual Differences 50(4), 492-495. doi:10.1016/j.paid.2010.11.013

Ursache, A., \& Raver, C. (2015). Iowa Gambling Task performance and executive function predict low-income urban preadolescents' risky behaviors. Personality and Individual Differences, 79, 1-6. doi:10.1016/j.paid.2015.01.010

van den Bos, R., Houx, B. B., \& Spruijt, B. M. (2006). The effect of reward magnitude differences on choosing disadvantageous decks in the Iowa Gambling Task. Biological Psychology, 71(2), 155-161. doi:10.1016/j.biopsycho.2005.05.003

van Honk, J., Hermans, E. J., Putman, P., Montagne, B., \& Schutter, D. J. L. G. (2002). Defective somatic markers in sub-clinical psychopathy. Neuroreport, 13(8), 1025-1027. Retirado de http://journals.lww.com/neuroreport/pages/default.aspx

van Honk, J., Schutter, D. J., Hermans, E. J., Putman, P., Tuiten, A., \& Koppeschaar, H. (2004). Testosterone shifts the balance between sensitivity for punishment and reward in healthy young women. Psychoneuroendocrinology, 29(7), 937-943. doi:10.1016/j.psyneuen.2003.08.007

Vanman, E. J., Mejia, V. Y., Dawson, M. E., Schell, A. M., \& Raine, A. (2003). Modification of the startle reflex in a community sample: Do one or two dimensions of psychopathy underlie emotional processing? Personality and Individual Differences, 35(8), 2007-2021. doi:10.1016/S0191-8869(03)00052-7

Vasconcelos, A., Sergeant, J., Corrêa, H., Mattos, P., \& MalloyDiniz, L. (2014). When self-report diverges from performance: The usage of BIS-11 along with neuropsychological tests. Psychiatry Research, 218(1-2), 236-243. doi:10.1016/j. psychres.2014.03.002

Vassileva, J., Georgiev, S., Martin, E., Gonzalez, R., \& Segala, L. (2011). Psychopathic heroin addicts are not uniformly impaired across neurocognitive domains of impulsivity. Drug and Alcohol Dependence, 114(2-3), 194-200. doi:10.1016/j. drugalcdep.2010.09.021
Vassileva, J., Gonzalez, R., Bechara, A., \& Martin, E. M. (2007). Are all drug addicts impulsive? Effects of antisociality and extent of multidrug use on cognitive and motor impulsivity. Addictive Behaviors, 32(12), 3071-3076. doi:10.1016/j. addbeh.2007.04.017

Walters, G. D. (2003). Predicting criminal justice outcomes with the Psychopathy Checklist and Life style Criminality Screening Form: A meta-analytic comparison. Behavioral Sciences and the Law, 21(1), 89-102. doi:10.1002/bsl.519

Wang, S., Krajbich, I., Adolphs, R., \& Tsuchiya, N. (2012). The role of risk aversion in non-conscious decision-making. Frontiers in Psychology, 3. doi:10.3389/fpsyg.2012.00050

Webb, C. A., DelDonno, S., \& Killgore, W. D. S. (2014). The role of cognitive versus emotional intelligence in Iowa Gambling Task performance: What's emotion got to do with it? Intelligence, 44, 112-119. doi:10.1016/j.intell.2014.03.008

Xu, S., Korczykowski, M., Zhu, S., Rao, H. (2013). Risk-taking and impulsive behaviors: A comparative assessment of three tasks. Social Behavior and Personality, 41(3), 477-486. doi:10.2224/ sbp.2013.41.3.477

Yamamoto, D. J., Woo, C.-W., Wager, T. D., Regner, M. F., \& Tanabe, J. (2015). Influence of dorsolateral prefrontal cortex and ventral striatum on risk avoidance in addiction: A mediation analysis. Drug and Alcohol Dependence, 149, 10-17. doi:10.1016/j.drugalcdep.2014.12.026

Yang, Y., Zhong, X., Wu, D., Li, H., \& Li, M. (2016). Positive association between trait impulsivity and high gambling-related cognitive biases among college students. Psychiatry Research, 243(30), 71-74. doi:10.1016/j.psychres.2016.04.110

Young, S., Gudjonsson, G. H., Goodwin, E, J., Derek, P., \& Robin, M. (2013). A validation of a computerised task of risk-taking and moral decision-making and its association with sensationseeking, impulsivity and sociomoral reasoning. Personality and Individual Differences, 55(8), 941-946. doi: 10.1016/j. paid.2013.07.472

Zhang, L., Wang, K., Zhu, C., Yu, F., \& Chen (2015). Trait anxiety has effect on decision making under ambiguity but not decision making under risk. PLoS One. 10(5), e0127189. doi:10.1371/ journal.pone.0127189

Zimak, E. H., Suhr, J., \& Bolinger, E. (2014). Psychophysiological and neuropsychological characteristics of non-incarcerated adult males with higher levels of psychopathic personality traits. Journal of Psychopathology and Behavioral Assessment, 36(4), 542-554. doi:10.1007/s 10862-014-9430-5 